\title{
Ex vivo model of non-small cell lung cancer using mouse lung epithelial cells
}

\author{
TAKU SATO $^{1-3}$, MAMI MORITA ${ }^{1}$, RYOTA TANAKA ${ }^{1-3}$, YUI INOUE $^{1}$, MIYUKI NOMURA $^{1}$, \\ YOSHIMI SAKAMOTO ${ }^{1}$, KOH MIURA ${ }^{4}$, SHIGEMI ITO ${ }^{1}$, IKURO SATO ${ }^{5}$, NOBUYUKI TANAKA ${ }^{6}$, \\ JIRO ABE ${ }^{2}$, SATOMI TAKAHASHI ${ }^{2}$, MASAAKI KAWAI $^{7}$, MASAMI SATO $^{8}$, YOSHITAKA HIPPO ${ }^{9}$, \\ HIROSHI SHIMA $^{1,10}$, YOSHINORI OKADA ${ }^{3}$ and NOBUHIRO TANUMA ${ }^{1,10}$
}

${ }^{1}$ Division of Cancer Chemotherapy, Miyagi Cancer Center Research Institute; ${ }^{2}$ Department of Thoracic Surgery, Miyagi Cancer Center Hospital, Natori, Miyagi 981-1293; ${ }^{3}$ Department of Thoracic Surgery, Institute of Development, Aging and Cancer, Tohoku University, Sendai, Miyagi 980-8575; ${ }^{4}$ Department of Surgery; ${ }^{5}$ Tissue Bank; ${ }^{6}$ Division of Cancer Biology and Therapeutics , Miyagi Cancer Center Research Institute; ${ }^{7}$ Department of Breast Oncology, Miyagi Cancer Center Research Institute, Natori, Miyagi 981-1293; ${ }^{8}$ Department of General Thoracic Surgery, Graduate School of Medical and Dental Sciences, Kagoshima University, Kagoshima, Kagoshima 890-8544;

${ }^{9}$ Division of Molecular Carcinogenesis, Chiba Cancer Center Research Institute, Chiba, Chiba 260-8717;

${ }^{10}$ Division of Cancer Molecular Biology, Tohoku University Graduate School of Medicine, Natori, Miyagi 981-1293, Japan

Received May 24, 2017; Accepted September 20, 2017

DOI: $10.3892 / 01.2017 .7098$

\begin{abstract}
Lung cancer is the most common cause of cancer mortality, however, efficient methods to culture, expand and transform lung epithelial (LE) cells have not been established. In the present study, an efficient ex vivo method was applied to recapitulate lung carcinogenesis using mouse LE cells. A Matrigel-assisted three-dimensional culture was used to isolate and selectively expand LE cells from mouse lungs. Purified LE cells were passaged and expanded for at least 2 to 3 months while maintaining epidermal growth factor-dependence. LE cells were also easily transformed by genetic manipulations using retroviral vectors. A SV40 large-T antigen, suppressing p53 and pRB, plus an activated oncogene, such as $\mathrm{Kras}^{\mathrm{G} 12 \mathrm{~V}}$ or EGFR ${ }^{\mathrm{ex} 19 \mathrm{del}}$, were required to transform LE cells. Transformed cells formed tumors resembling non-small cell lung cancer (NSCLC) in allograft models and exhibited strong oncogene addiction. This experimental system provided a unique model system to study lung tumorigenesis and develop novel therapeutics against NSCLC.
\end{abstract}

Correspondence to: Dr Nobuhiro Tanuma, Division of Cancer Chemotherapy, Miyagi Cancer Center Research Institute, 47 Noda-yama, Medeshima-shiode, Natori, Miyagi 981-1293, Japan E-mail: ntanuma@med.tohoku.ac.jp

Key words: lung cancer, tumorigenesis, Kras, EGFR, non-small cell lung cancer

\section{Introduction}

Lung cancer is the most common cause of cancer mortality in the world, with non-small cell lung cancer (NSCLC) its most prevalent form (1). Despite recent development of oncogene-targeting therapy and immunotherapy, the prognosis for NSCLC at advanced stages remains poor $(2,3)$. Either chemically-induced or genetically-engineered mouse models, as well as patient-derived xenograft models, have provided preclinical models to understand NSCLC and to test novel therapeutic approaches (4). Nonetheless, more efficient methods to culture, expand and transform lung epithelial (LE) cells are required.

The linings of organs are constructed from epithelial tissues, which are the origin of most of solid malignancies. Epithelial cells are connected to each other and/or with the basement membrane, and these cell-cell or cell-matrix interactions cannot be completely recapitulated in $2 \mathrm{D}$ culture, rendering analysis difficult using non-transformed epithelial cells in vitro and ex vivo. But recent advances in three-dimensional (3D) culture techniques have enabled in vitro expansion, genetic or environmental manipulation and real-time observations of epithelial cells from numerous human and rodent organs $(5,6)$.

In this study, we applied a 3D culture technique to isolate epithelial cells from a mixed population of mouse lung cells. Almost all isolated cells were positive for EpCAM, an epithelial surface-marker. LE cells could be passaged and expanded for several months and were easily transformed by genetic manipulation.

\section{Materials and methods}

Materials. Human EGFR cDNA encoding the exon 19-deletion mutant $\left(\mathrm{EGFR}^{\mathrm{ex} 19 \mathrm{del}}\right)$ was generated by a 
polymerase chain reaction-based method (7) and subcloned into a retroviral vector, pMXs-Puro-3xHA, as described previously (7). The plasmid pBabe-puro Kras-V12 was a gift from Dr C. Counter (8). The plasmid pBabe-neo largeT cDNA was a gift from Dr R. Weinberg (9). Matrigel was obtained from BD Biosciences (Franklin Lakes, NJ, USA). Murine EGF (mEGF) was purchased from PeproTech Inc. (Rocky Hill, NJ, USA). Erlotinib was purchased from Wako Pure Chemical Industries (Osaka, Japan). Trametinib and Captisol were purchased from ChemScene (Monmouth Junction, NJ, USA). Y-27632 was purchased from Calbiochem (Darmstadt, Germany). TrypLE Express from Thermo Fisher Scientific Inc. (Waltham, MA, USA).

Isolation and 3D culture of mouse LE cells. C57BL/6 mice were used at 6-9 weeks of age. Isolation, 3D culture and passage were performed similarly to methods previously described for colon epithelial cells, except that R-Spondin, Noggin and Jagged-1 were not added to culture media (10). In various analyses, mEGF and Y-27632 were added to the medium both in 3D and 2D culture, both at final concentrations of $50 \mathrm{nM}$. To passage cells in 2D culture, cells were washed with PBS, treated with Trypl Express reagent, which is a mixture of protease and collagenase, and then detached from the dish using a cell-scraper. Time-lapse imaging was performed using an Incucyte cell-analyzer (Essen Bioscience, Ann Arbor, MI, USA).

Caspase activity assay. Cellular caspase activity was measured using the Caspase-Glo 3/7 Assay kit according to the manufacturer's recommendation (Promega, Madison, WI, USA). The values were normalized to cell numbers. The results shown in Fig. 2C is results from analyses of cells $2 \mathrm{~h}$ after the re-plating upon passage.

Infection of LE cells with retrovirus. LE cells were transduced with retroviral vectors harboring $\mathrm{Kras}^{\mathrm{G} 12 \mathrm{~V}}$, EGFR ${ }^{\mathrm{ex} 19 \mathrm{del}}$, or SV40 Large-T plus selection marker $\left(\right.$ Puro $^{\mathrm{r}}$ for $\mathrm{Kras}^{\mathrm{G} 12 \mathrm{~V}}$ and $\mathrm{EGFR}^{\mathrm{ex} 19 \mathrm{del}}$, and $\mathrm{Neo}^{\mathrm{r}}$ for SV40 Large-T construct). Ecotropic viruses were packaged using PLAT-E cells (a gift from Dr T. Kitamura, Tokyo University) (11) and Fugene 6 transfection reagent. Infection of LE cells in $3 \mathrm{D}$ culture with retroviruses was performed as described (12).

Animal experiments. All animal experiments were performed after approval of Miyagi Cancer Center Research Institute Animal Care and Use committee. In allograft experiments, $4 \times 10^{5}$ of LE-LT/Kras ${ }^{\mathrm{G} 12 \mathrm{~V}}$ cells were injected $s c$ into the dorsal flank of nude mice. LE-LT/EGFR ${ }^{\text {ex19del }}$ cells were injected as 1:1 mixtures with Matrigel at $2 \times 10^{6}$ cells per injection site.

Immunostaining analyses and flow cytometry. Immunohistochemical analyses were performed using reagents from Roche Ventana systems (Basel, Switzerland). Antibodies against thyroid transcription factor 1 (TTF1) (SP141) and Ki-67 (30-9) were from Roche. Anti-cytokeratin 14 (CK14) goat antibody was purchased from Santa Cruz Biotechnology (Dallas, TX, USA). Anti-prosurfactant protein-C (SPC) antibody (ab40879) was obtained from Abcam (Cambridge, UK). Anti-EpCAM monoclonal antibody
(2-17-F-1) was obtained from MBL (Nagoya, Japan). Frozen sections were prepared for anti-EpCAM staining only, as the antibody did not work on paraffin-embedded sections (data not shown). Flow cytometry was performed with a FACS Canto-II flow cytometer (BD Bioscences Inc.).

Statistics. We used Student's t-test (2-tailed) to compare two groups. A P-value of $<0.05$ was considered significant. Data are presented as means with the SD (Fig. 2B and D) or SEM (all others).

\section{Results}

Isolation and characterization of primary LE cells. Primary LE cells were isolated from adult mice and expanded in Matrigel-assisted 3D culture for 2 to 3 weeks in the presence of EGF (Fig. 1A and B). During this period, non-epithelial cells, such as fibroblasts, were likely lost or selected against, as nearly all expanded cells were positive for EpCAM, an established marker of the epithelial lineage (Fig. 1C) (13). In $3 \mathrm{D}$ culture, LE cells could be propagated, for at least two to three months, with passage once weekly at an approximately 1:4 dilution.

To test EGF-dependence of LE cells, we transferred cells to 2D culture, as Matrigel used for 3D culture contains substantial amounts of EGF (14). Then, we prepared single-cell suspensions from LE spheres formed in $3 \mathrm{D}$ culture and seeded them in tissue-culture plates in the presence of EGF and the Rock-inhibitor Y-27632. Cells adapted to 2D culture (Fig. 2A) and were passaged (1:5 dilution) several times, always replacing EGF and Y-27632 in the media ('Control' in Fig. 2B and C). We confirmed that LE cells passaged in 2D were still EpCAM-positive. In addition, the 2D-cultured cells as monolayer were converted into 3D-spheres again when re-transferred on Matrigel. To evaluate the requirement for either of these factors, we prepared replicates of culture when cells were passaged and then withdrew EGF or Y-27632 from the media the next day. Upon removing EGF, LE cells in $2 \mathrm{D}$ culture ceased proliferating and became more flattened and enlarged (Fig. 2A), exhibiting the morphology of senescent cells, and died after the next passage (Fig. 2B). Upon the passage, the LE cells deprived of EGF showed markedly higher activity of caspase, suggesting apoptosis induction, compared to control cells (Fig. 2C). We also observed that the presence of both EGF and the Rock-inhibitor was required for maximal LE cell proliferation in 2D culture (Fig. 2D), as Y-27632 withdrawal immediately decreased cell proliferation. These results overall indicate that LE cells expanded in $3 \mathrm{D}$ culture over a long period maintain their requirement for EGF to proliferate.

LE cell transformation requires both oncogene activation and tumor-suppressor loss. We next transformed LE cells by transduction with SV40 Large-T and the active Kras-mutant $\mathrm{Kras}^{\mathrm{G} 12 \mathrm{~V}}$, either singly or together, using retroviral vectors harboring $\mathrm{NeO}^{r}$ and $\mathrm{Puro}^{r}$, respectively, as selection marker. Transduced cells were then drug-selected and nude mice were inoculated with drug-resistant cells. Only LE cells receiving both Large-T and $\mathrm{Kras}^{\mathrm{G} 12 \mathrm{~V}}$ (LE-LT/ $\mathrm{Kras}^{\mathrm{G} 12 \mathrm{~V}}$ cells) formed allograft tumors, while cells harboring either Large-T 


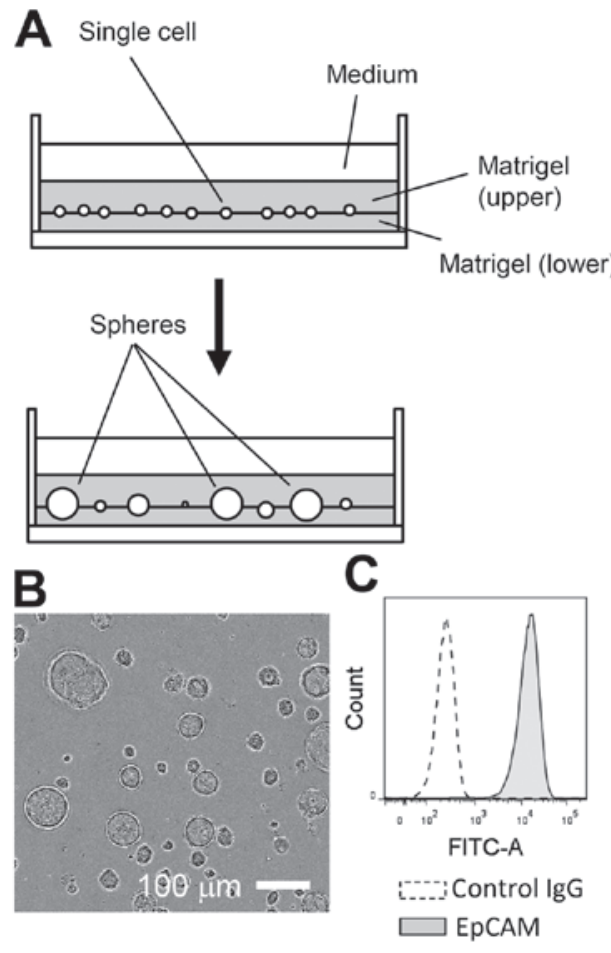

Figure 1. Long-term 3D culture of primary epithelial cells isolated from murine lung. (A) Schematic showing LE cell expansion in Matrigel-embedded 3D culture. (B) Photomicrographs of LE spheres. (C) LE cells were stained with anti-EpCAM antibody and analyzed by flow cytometry. Staining with control $\mathrm{Ab}(\mathrm{rat} \mathrm{IgG})$ served as negative control. LE, lung epithelial; EpCAM, epithelial cell adhesion molecule precursor.

or $\mathrm{Kras}^{\mathrm{G} 12 \mathrm{~V}}$ alone did not (Fig. 3A and B). These results are consistent with previous observations that cellular transformation requires both oncogene activation and inactivation of tumor suppressors such as p53 and pRb (15).

Immunostaining of LE-LT/ Kras ${ }^{\mathrm{G} 12 \mathrm{~V}}$ tumor showed that the Ki-67 index of the tumor was very high ( $25 \%$; Fig. $3 \mathrm{C})$ and that tumor cells arising from LE cells were mostly EpCAM-positive (Fig. 3D). In addition, a part of tumor cells were positive for TTF1, an established marker of pulmonary adenocarcinoma (Fig. 3E). No staining in tumors was observed for SP-C and CK14, respective markers of lung adenocarcinoma and squamous-cell carcinoma (data not shown). Together with H\&E analysis (Fig. 3B), these results suggest that tumors derived from LE-LT/ $\mathrm{Kras}^{\mathrm{G} 12 \mathrm{~V}}$ cells are largely anaplastic (expressing neither adenocarcinoma marker nor squamous-cell carcinoma marker) and that a subpopulation of tumor cells tends to differentiate into adenocarcinoma.

In addition to $\mathrm{Kras}^{\mathrm{G} 12 \mathrm{~V}}$, we also transformed LE cells with EGFR $^{\text {ex19del }}$, the constitutively active mutant of EGFR, in combination with Large-T. Like the LE-LT/Kras ${ }^{\mathrm{G} 12 \mathrm{~V}}$ cells, the LE-LT/EGFR ${ }^{\text {ex } 19 \text { del }}$ cells formed tumors when transplanted into nude mice (data not shown).

Oncogene addiction of transformed LE cells. It is well known that tumorigenicity due to $\mathrm{Kras}^{\mathrm{Gi2V}}$ activity requires activation of the classical mitogen-activated protein kinase (MAPK) cascade, in which MAPK/ERK kinase (MEK) phosphorylates and activates extracellular regulated kinase (Erk) (16). Proliferation of

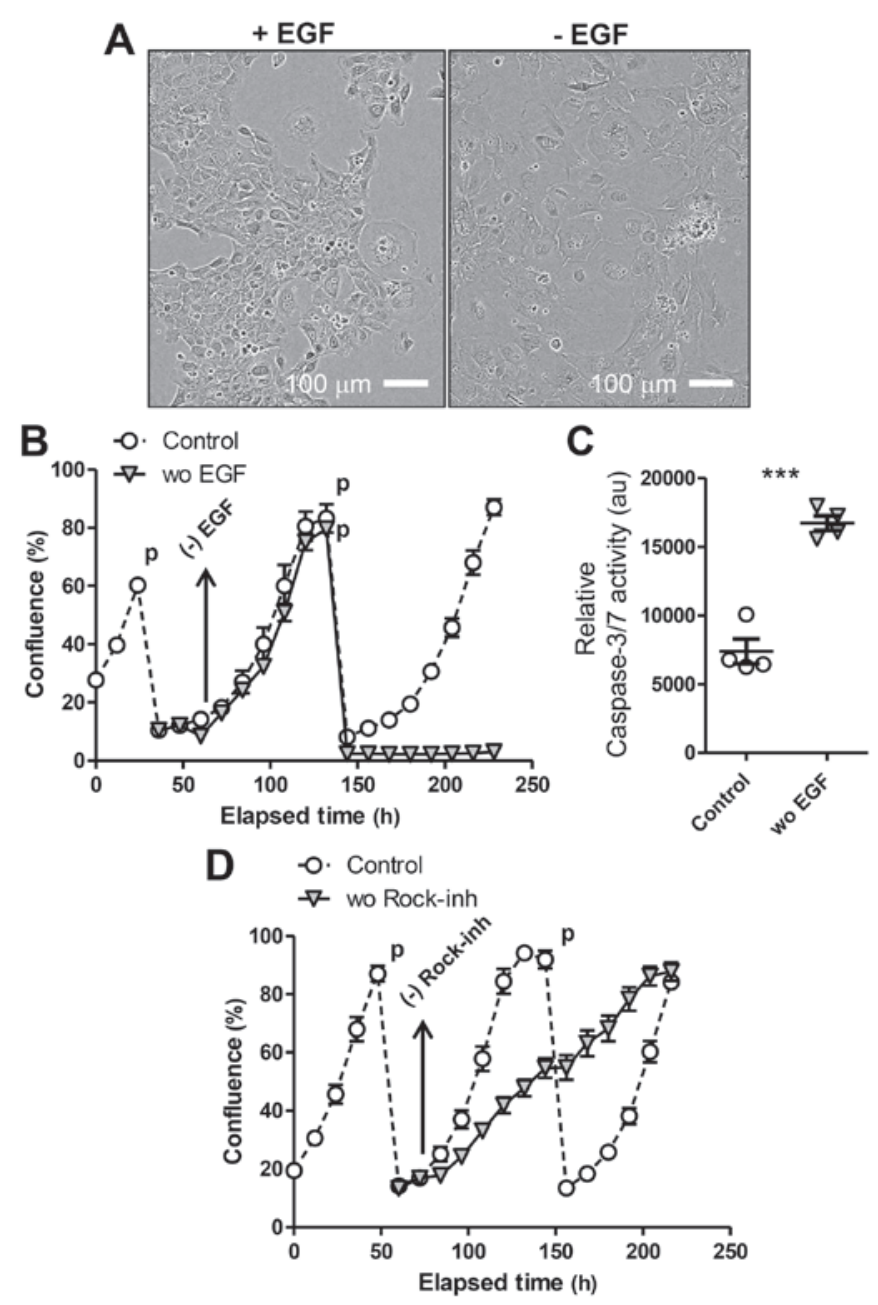

Figure 2. EGF-dependence of LE cells. (A) Photomicrographs of LE cells in 2D culture in the presence (upper) or absence (lower) of EGF. (B) Time-lapse analysis of LE cell proliferation over the culture period. 'p' indicates that cells were passaged at that point. Timing of EGF-withdrawal is shown by arrow. (C) Caspase-3/7 activity of cells cultured with or without EGF and passaged as in $\mathrm{B} .{ }^{* * *} \mathrm{P}=0.0001$ vs. control; two-tailed t-test. (D) Effect of withdrawal of Y-27632 on proliferation of LE cells in 2D culture as analyzed in B. EGF, epidermal growth factor; LE, lung epithelial.

LE-LT/Kras ${ }^{\mathrm{G} 12 \mathrm{~V}}$ cells in vitro was independent of exogenous EGF (data not shown), which activates the MEK-ERK pathway. We then tested the sensitivity of LE-LT/Kras ${ }^{\mathrm{G} 12 \mathrm{~V}}$ cells in vitro to trametinib, a MEK1-inhibitor. As shown in Fig. 4A, proliferation of LE-LT/Kras ${ }^{\mathrm{G} 12 \mathrm{~V}}$ cells markedly decreased following trametinib treatment, whereas comparable treatment with erlotinib, which potently inhibits EGFR kinase activity, had no effect. In contrast, both trametinib and erlotinib treatment inhibited LE-LT/EGFR ${ }^{\text {ex19del }}$ cell proliferation (Fig. 4B). These results are consistent with the idea that Kras activates MAPK signaling downstream of EGFR, making cells resistant to EGFR inhibition and that transformation by $\mathrm{EGFR}^{\mathrm{ex} 19 \mathrm{del}}$ requires its kinase activity and downstream MAPK signaling (17). Overall, these results show that transformed LE cells are addicted to oncogenic Kras- or EGFR-stimulated signaling.

\section{Discussion}

Use of 3D-culture has become a powerful tool for development of tissue engineering methods for regenerative medicine 

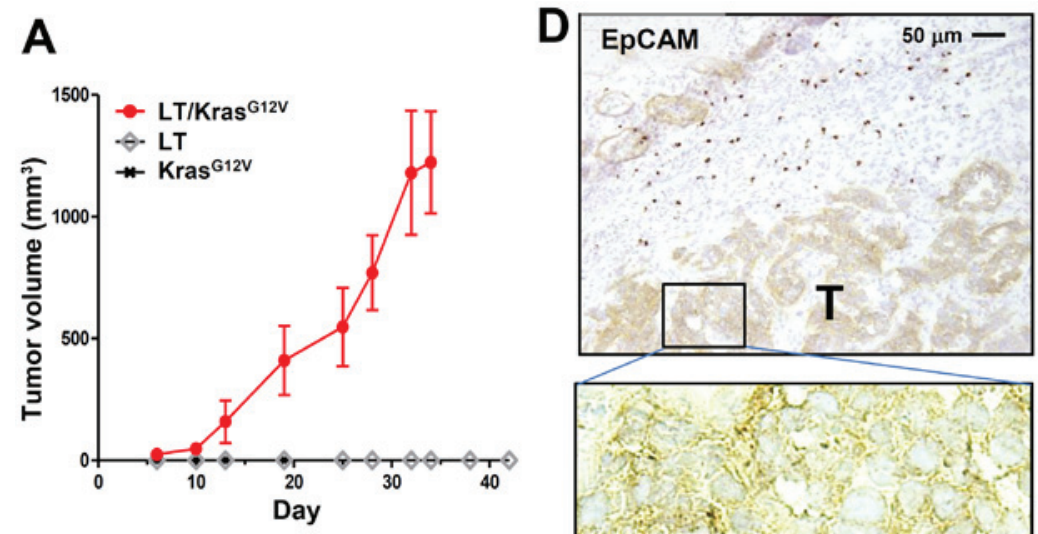

\section{B}

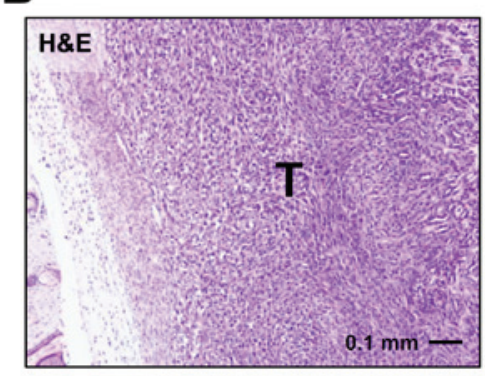

C
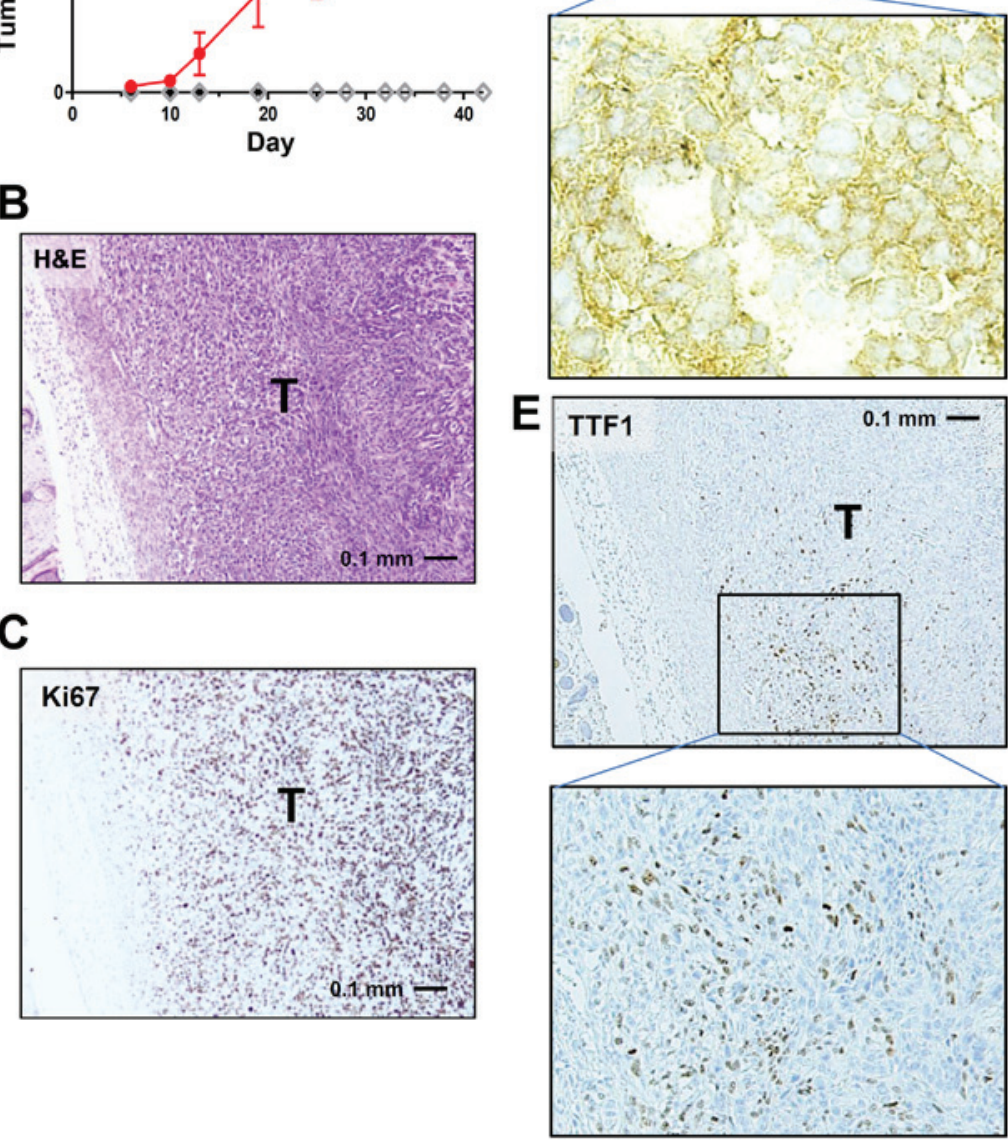

Figure 3. LE cell transformation with SV40 large-T and oncogenic-Kras. (A) LE cells were infected with retrovirus expressing either SV40 large-T (LT) or $\mathrm{Kras}^{\mathrm{G} 12 \mathrm{~V}}$ or both. Drug-selected cells were then inoculated sc into nude mice. Tumor volume was monitored for $\sim 40$ days. (B) H\&E staining of a tumor derived from LE-LT/Kras ${ }^{\mathrm{G} 12 \mathrm{~V}}$ cells. T indicates tumor. (C-E) Sections of tumors of LE-LT/Kras ${ }^{\mathrm{G} 12 \mathrm{~V}}$ cells stained with anti-Ki67 (C), anti-EpCAM (D) or anti-TTF1 (E) antibodies. T indicates tumor. LE, lung epithelial; H\&E, hematoxylin and eosin; TTF1, thyroid transcription factor 1.
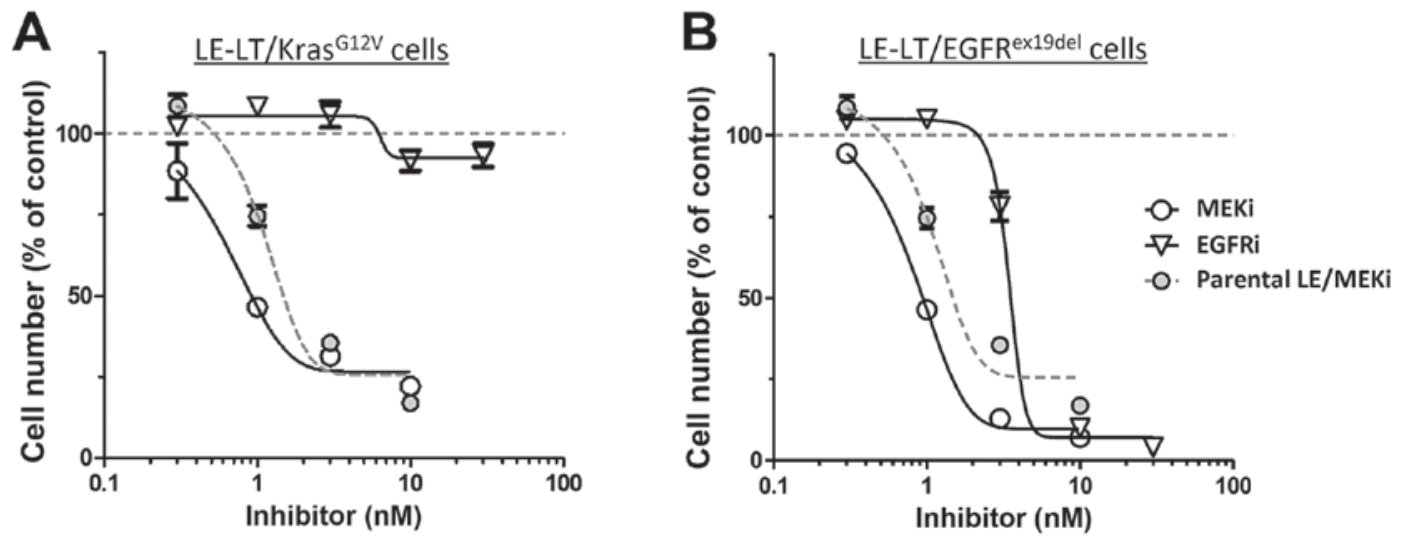

Figure 4. Oncogene addiction of transformed LE cells. (A) LE-LT/Kras ${ }^{\mathrm{Gi2}}$ cells cultured in vitro were treated with the MEKi (trametinib) or the EGFRi (erlotinib) for 2 days at concentrations indicated. Cell growth was quantified using sulpho-rhodamine B staining. The MEKi-sensitivity of parental LE cells (4 days treatment) are also shown for comparison. Results are shown relative to vehicle-treated control cells. (B) LE-LT/EGFR ${ }^{\text {ex19del }}$ cells were treated with MEKi or EGFRi for 4 days and analyzed as in A. Data shown are means with SEM. LE, lung epithelial; EGF, epidermal growth factor receptor; MEKi, mitogen-activated protein kinase/extracellular signal-regulated kinase inhibitor. 
and to understand cancer biology $(6,18)$. Relevant to the latter, in colorectal cancer research, use of 3D culture has revealed the identity of cancer cells of origin, the function of Yap-dependent regenerative signaling in cancer initiation, and how niche factor requirements are lost during colon cancer progression $(19,20)$. Given the utility of 3D culture in expanding normal epithelial cells, we used this method here to isolate, expand, and transform mouse LE cells.

While our method could be applied to numerous experimental settings, one of particular interest is analysis of cells from genetically-engineered mice in several ways. For example, although several sophisticated mouse models of NSCLC have been established in the last decade (4), it is time-consuming to obtain mice carrying the multiple mutant alleles required for analyses, often requiring a year or more. Our method yields either transformed LE cells or NSCLC-like allograft tumors normally within 2 or 3 months, respectively.

Pharmacological experiments using MEK and/or EGFR inhibitors show that proliferation of transformed LE cells is highly dependent on signaling by the transduced oncogene. Thus, our system could be useful to identify novel chemo-preventatives and therapeutics and to test their efficacy in vitro or in transplantation models. Also, allograft models using syngeneic strains will enable experiments in immune-competent mice, providing a unique opportunity to explore novel immunotherapy approaches and pre-clinical models for testing their efficacy (21).

Tumors arising from transformed mouse LE cells are largely anaplastic. It may be possible to create more differentiated tumors using different combination(s) of oncogenes and tumor-suppressor inhibitions. Alternatively, more differentiated tumors may be achieved using environmental cues, as it is well known that specific culture conditions are critical to promote induction of specific lineages from stem cells (22). To date, several cell types found in the pulmonary system, such as alveolar type 2 cells, club cells, and tracheobronchial basal cells, have been reported as NSCLC cells of origin $(13,23)$. Accordingly, detailed characterization such as comprehensive marker and transcriptome analyses are needed to accurately define identities of LE cells cultured long-term.

In summary, we established an ex vivo method to recapitulate tumorigenesis of mouse LE cells. Our experimental system provides a unique opportunity to study lung tumorigenesis and to develop novel therapeutics against NSCLC. Characterization of long-term expanded LE cells also has implications for regenerative approaches to lung disease, such as chronic obstructive pulmonary disease (24).

\section{Acknowledgements}

We would like to acknowledge Dr C. Counter and Dr R. Weinberg for providing plasmid constructs. Thanks are also due to members of the Pathology facility of Miyagi Cancer Center Hospital for technical help in tissue analyses, and to Y. Chiba for secretarial assistance. The present study was supported by grants from JSPS KAKENHI grants (16K14621) to N. T., 26430130 to H. S., 16K07187 to S. I., $15 \mathrm{~K} 14387$ to I. S., and $16 \mathrm{~K} 10486$ to K. M.), and to the Takeda
Foundation (N. T.), the Mochida Memorial Foundation for Medical and Pharmaceutical Research (N. T.), the Kato Memorial Bioscience Foundation (N. T.), the Uehara Memorial Foundation (N. T.) and the Sagawa Foundation for Promotion of Cancer Research (N. T.).

\section{References}

1. Chen Z, Fillmore CM, Hammerman PS, Kim CF and Wong KK: Non-small-cell lung cancers: A heterogeneous set of diseases. Nat Rev Cancer 14: 535-546, 2014.

2. Sundar R, Cho BC, Brahmer JR and Soo RA: Nivolumab in NSCLC: Latest evidence and clinical potential. Ther Adv Med Oncol 7: 85-96, 2015.

3. Maemondo M, Inoue A, Kobayashi K, Sugawara S, Oizumi S, Isobe H, Gemma A, Harada M, Yoshizawa H, Kinoshita I, et al: Gefitinib or chemotherapy for non-small-cell lung cancer with mutated EGFR. N Engl J Med 362: 2380-2388, 2010.

4. DuPage M, Dooley AL and Jacks T: Conditional mouse lung cancer models using adenoviral or lentiviral delivery of Cre recombinase. Nat Protoc 4: 1064-1072, 2009.

5. Shamir ER and Ewald AJ: Three-dimensional organotypic culture: Experimental models of mammalian biology and disease. Nat Rev Mol Cell Biol 15: 647-664, 2014.

6. Clevers H: Modeling development and disease with organoids. Cell 165: 1586-1597, 2016.

7. Watanuki Z, Kosai H, Osanai N, Ogama N, Mochizuki M Tamai K, Yamaguchi K, Satoh K, Fukuhara T, Maemondo M, et al: Synergistic cytotoxicity of afatinib and cetuximab against EGFR T790M involves Rab11-dependent EGFR recycling. Biochem Biophys Res Commun 455: 269-276, 2014.

8. Lampson BL, Pershing NL, Prinz JA, Lacsina JR, Marzluff WF, Nicchitta CV, MacAlpine DM and Counter CM: Rare codons regulate KRas oncogenesis. Curr Biol 23: 70-75, 2013.

9. Hahn WC, Dessain SK, Brooks MW, King JE, Elenbaas B, Sabatini DM, DeCaprio JA and Weinberg RA: Enumeration of the simian virus 40 early region elements necessary for human cell transformation. Mol Cell Biol 22: 2111-2123, 2002.

10. Onuma K, Ochiai M, Orihashi K, Takahashi M, Imai T, Nakagama $\mathrm{H}$ and Hippo Y: Genetic reconstitution of tumorigenesis in primary intestinal cells. Proc Natl Acad Sci USA 110: 11127-11132, 2013.

11. Morita S, Kojima T and Kitamura T: Plat-E: An efficient and stable system for transient packaging of retroviruses. Gene Ther 7: 1063-1066, 2000.

12. Tanuma N, Nomura M, Ikeda M, Kasugai I, Tsubaki Y, Takagaki K, Kawamura T, Yamashita Y, Sato I, Sato M, et al: Protein phosphatase Dusp26 associates with KIF3 motor and promotes N-cadherin-mediated cell-cell adhesion. Oncogene 28: 752-761, 2009.

13. Asselin-Labat ML and Filby CE: Adult lung stem cells and their contribution to lung tumourigenesis. Open Biol 2: 120094, 2012.

14. Hughes CS, Postovit LM and Lajoie GA: Matrigel: A complex protein mixture required for optimal growth of cell culture. Proteomics 10: 1886-1890, 2010.

15. Vogelstein B, Papadopoulos N, Velculescu VE, Zhou S, Diaz LA Jr and Kinzler KW: Cancer genome landscapes. Science 339: 1546-1558, 2013.

16. Katz M, Amit I and Yarden Y: Regulation of MAPKs by growth factors and receptor tyrosine kinases. Biochim Biophys Acta 1773: 1161-1176, 2007.

17. Sharifnia T, Rusu V, Piccioni F, Bagul M, Imielinski M, Cherniack AD, Pedamallu CS, Wong B, Wilson FH, Garraway LA, et al: Genetic modifiers of EGFR dependence in non-small cell lung cancer. Proc Natl Acad Sci USA 111: 18661-18666, 2014.

18. Fatehullah A, Tan SH and Barker N: Organoids as an in vitro model of human development and disease. Nat Cell Biol 18: 246-254, 2016.

19. Gregorieff A, Liu Y, Inanlou MR, Khomchuk Y and Wrana JL: Yap-dependent reprogramming of Lgr5(+) stem cells drives intestinal regeneration and cancer. Nature 526: 715-718, 2015

20. Fujii M, Shimokawa M, Date S, Takano A, Matano M, Nanki K, Ohta Y, Toshimitsu K, Nakazato Y, Kawasaki K, et al: A colorectal tumor organoid library demonstrates progressive loss of niche factor requirements during tumorigenesis. Cell Stem Cell 18: 827-838, 2016. 
21. Akbay EA, Koyama S, Carretero J, Altabef A, Tchaicha JH, Christensen CL, Mikse OR, Cherniack AD, Beauchamp EM, Pugh TJ, et al: Activation of the PD-1 pathway contributes to immune escape in EGFR-driven lung tumors. Cancer Discov 3 : $1355-1363,2013$.

22. Huang SX, Islam MN, O'Neill J, Hu Z, Yang YG, Chen YW, Mumau M, Green MD, Vunjak-Novakovic G, Bhattacharya J and Snoeck HW: Efficient generation of lung and airway epithelial cells from human pluripotent stem cells. Nat Biotechnol 32: 84-91, 2014.
23. Desai TJ, Brownfield DG and Krasnow MA: Alveolar progenitor and stem cells in lung development, renewal and cancer. Nature 507: 190-194, 2014

24. Kotton DN and Morrisey EE: Lung regeneration: Mechanisms, applications, and emerging stem cell populations. Nat Med 20: 822-832, 2014. 